\title{
A Parallel-Cone Collimator for High-Energy SPECT
}

\author{
Casper Beijst ${ }^{1,2}$, Mattijs Elschot ${ }^{1}$, Max A. Viergever ${ }^{2}$, and Hugo W.A.M de Jong ${ }^{1}$ \\ ${ }^{1}$ Radiology and Nuclear Medicine, UMC Utrecht, Utrecht, The Netherlands; and ${ }^{2}$ Image Sciences Institute, UMC Utrecht, Utrecht, \\ The Netherlands
}

In SPECT using high-energy photon-emitting isotopes, such as ${ }^{131}$, parallel-hole collimators with thick septa are required to limit septal penetration, at the cost of sensitivity and resolution. This study investigated a parallel-hole collimator with cone-shaped holes, which was designed to limit collimator penetration while preserving resolution and sensitivity. The objective was to demonstrate that a singleslice prototype of the parallel-cone $(P C)$ collimator was capable of improving the image quality of high-energy SPECT. Methods: The image quality of the PC collimator was quantitatively compared with that of clinically used low-energy high-resolution (LEHR; for ${ }^{99 m T C)}$ and high-energy general-purpose (HEGP; for ${ }^{131} \mathrm{I}$ and ${ }^{18} \mathrm{~F}$ ) parallel-hole collimators. First, Monte Carlo simulations of single and double point sources were performed to assess sensitivity and resolution by comparing point-spread functions (PSFs). Second, a prototype PC collimator was used in an experimental phantom study to assess and compare contrast recovery coefficients and image noise. Results: Monte Carlo simulations showed reduced broadening of the PSF due to collimator penetration for the PC collimator as compared with the HEGP collimator (e.g., 0.9 vs. $1.4 \mathrm{~cm}$ in full width at half maximum for $\left.{ }^{131} \mathrm{I}\right)$. Simulated double point sources placed $2 \mathrm{~cm}$ apart were separately detectable for the PC collimator, whereas this was not the case for ${ }^{131} \mathrm{I}$ and ${ }^{18} \mathrm{~F}$ at distances from the collimator face of $10 \mathrm{~cm}$ or more for the HEGP collimator. The sensitivity, measured over the simulated profiles as the total amount of counts per decay, was found to be higher for the LEHR and HEGP collimators than for the PC collimator (e.g., $3.1 \times 10^{-5}$ vs. $2.9 \times 10^{-5}$ counts per decay for $\left.{ }^{131} \mathrm{I}\right)$. However, at equal noise level, phantom measurements showed that contrast recovery coefficients were similar for the PC and LEHR collimators for ${ }^{99 \mathrm{mT}} \mathrm{T}$ but that the PC collimator significantly improved the contrast recovery coefficients as compared with the HEGP collimator for ${ }^{131} \mathrm{I}$ and ${ }^{18} \mathrm{~F}$. Conclusion: High-energy SPECT imaging with a single-slice prototype of the proposed PC collimator has shown the potential for significantly improved image quality in comparison with standard parallel-hole collimators.

Key Words: SPECT; high-energy SPECT; collimator; parallel cone collimator

J Nucl Med 2015; 56:476-482

DOI: 10.2967/jnumed.114.149658

\section{$\mathbf{S}$}

PECT imaging using isotopes emitting high-energy gamma or bremsstrahlung photons has important applications in oncology and is used for both diagnosis and therapy monitoring. Examples

Received Oct. 8, 2014; revision accepted Jan. 14, 2015.

For correspondence or reprints contact: Casper Beijst, University Medical Center Utrecht, Mail E01.132, P.O. Box 85500, 3508GA Utrecht, The Netherlands.

E-mail: cbeijst@umcutrecht.nl

Published online Feb. 5, 2015.

COPYRIGHT (c) 2015 by the Society of Nuclear Medicine and Molecular Imaging, Inc.

of applications include radioisotope therapy (e.g., ${ }^{131} \mathrm{I},{ }^{188} \mathrm{Re}$, and $\left.{ }^{67} \mathrm{Ga}\right)$, monitoring of antibodies $\left({ }^{111} \mathrm{In}\right)$, and internal radiation therapy $\left({ }^{90} \mathrm{Y}\right.$ and $\left.{ }^{166} \mathrm{Ho}\right)(1-4)$. SPECT images are used for qualitative and quantitative purposes, both requiring high image resolution and low image noise. SPECT imaging of these high-energy photon-emitting isotopes remains a challenge, because broadening of the point-spread function (PSF) due to penetration of collimator septa by high-energy photons severely degrades image quality $(5,6)$. Improvement of the quality of high-energy SPECT images is an important field of interest, which may have great impact on planning, follow-up, and dosimetry in cancer treatment (7).

The septal thickness, length, and hole size of parallel-hole collimators can be optimized for a specific application. The strategy used to optimize parallel-hole collimators for high-energy applications is to increase septal thickness and length to limit septal penetration. Over the years, several high-energy collimators have been designed to accommodate photon energies of 200-511 $\mathrm{keV}$, including the special high-energy all-purpose collimator (8), the ultra-high-energy collimator (9), and the more common highenergy general-purpose (HEGP) collimator. In general, increasing the septal thickness will decrease the amount of penetrated photons, but it will also decrease the system sensitivity. A decrease in sensitivity can be compensated for by increasing the hole size, but this will in turn compromise spatial resolution. In other words, optimizing the design of a parallel-hole collimator for high-energy SPECT involves a tradeoff between septal penetration, spatial resolution, and sensitivity.

Pinhole collimators are an interesting alternative to parallelhole collimators for high-energy SPECT imaging, because pinholes can be designed in such a way that collimator penetration is limited (7). Depending on the application and geometry, however, pinhole systems have either limited sensitivity or a small field of view (FOV); the latter is used, for example, in small-organ (10) and small-animal imaging (11). By combining the advantages of parallel-hole collimators and high-energy pinhole systems, we aimed to design a collimator with high sensitivity, high spatial resolution, and limited collimator penetration.

The objective of this work was to present and evaluate a collimator that yielded SPECT images for isotopes in the highenergy range that can replace a parallel-hole collimator in standard clinical SPECT systems without technical modifications. To this end, we proposed a collimator design (the parallel-cone $[\mathrm{PC}]$ collimator) consisting of a repetitive grid of parallel cones, which combined high spatial resolution and low septal penetration with high sensitivity, as compared with a parallel-hole collimator. Monte Carlo simulations and phantom experiments were performed with low-energy $\left({ }^{99 \mathrm{~m}} \mathrm{Tc}\right)$ and high-energy $\left({ }^{131} \mathrm{I}\right.$ and $\left.{ }^{18} \mathrm{~F}\right)$ isotopes, to quantitatively evaluate the performance of the PC collimator in comparison with standard clinical low-energy and high-energy parallel-hole collimators. In addition to ${ }^{131} \mathrm{I},{ }^{99 \mathrm{~m}} \mathrm{Tc}$ and ${ }^{18} \mathrm{~F}$ were 
included to gain insight into the performance of the PC collimator in situations without and with significant septal penetration.

\section{MATERIALS AND METHODS}

\section{Collimator Design}

Figure 1 shows a rendering of the PC collimator, consisting of multiple cones with a hexagonally shaped base that can be mounted on a standard gamma camera. The PC collimator was designed in such a way that collimator penetration by high-energy gamma photons is limited. Each cone's top diameter (pinhole diameter) is $4 \mathrm{~mm}$, and the cone base diameter (shortest distance across hexagonal base) is $40 \mathrm{~mm}$; its height is $100 \mathrm{~mm}$. The top of each cone is circular, but the bottom is hexagonal so as to have uniform septal thickness at the collimator bottom. The shape can be characterized as a single-sided knife-edge pinhole, which allows for small pinhole-to-object distances. If the PC collimator was made from lead in a size typical for most gamma cameras $(\sim 500 \times 400 \mathrm{~mm})$, the weight of the collimator would be approximately $180 \mathrm{~kg}$. The low-energy and high-energy parallel-hole collimators used in this study weighed 20.4 and $134.5 \mathrm{~kg}$, respectively.

The PC collimator performance was compared with the low-energy and high-energy parallel-hole collimators that are commonly used for SPECT imaging in clinical practice. The BiCore low-energy highresolution (LEHR) collimator (Siemens Healthcare) has 148,000 holes, a hole length of $24.05 \mathrm{~mm}$, a hole diameter of $1.11 \mathrm{~mm}$, and a septal thickness of $0.16 \mathrm{~mm}$. The BiCore HEGP collimator (Siemens Healthcare) has 8,000 holes, a hole length of $59.7 \mathrm{~mm}$, a hole diameter of $4.0 \mathrm{~mm}$, and a septal thickness of $2.0 \mathrm{~mm}$ (12).

\section{Prototype Collimator}

A prototype with a limited FOV for single-slice reconstructions was built, consisting of 6 cones positioned in a row, with the bases of adjacent cones placed $5 \mathrm{~mm}$ apart (Fig. 2; Supplemental Fig. 1 [supplemental materials are available at http://jnm.snmjournals.org]). The collimator was manufactured by lead casting (Nuclear Fields BV). The back side of the collimator was mounted on the crystal with 1-mm spacing, so that the distance of the collimator face to the crystal was $10.1 \mathrm{~cm}$.

\section{Monte Carlo Simulations}

To study the influence of collimator penetration on image quality, Monte Carlo simulations were performed using the Monte Carlo radiation transport code MCNPX 2.5.0 (13). MCNPX standard particle physics settings were used, and whenever the energy of a photon fell below $49 \mathrm{keV}$, it was terminated, simulating $5 \times 10^{7}$ particles. The Monte Carlo simulations took into account the photoelectric effect, coherent and Compton scattering, and bremsstrahlung and x-ray production by electrons. No variance reduction techniques were used. The input photon-energy spectra used for the MCNPX simulations were obtained from the nuclear data sheets (14). Photons and particles were tracked, and the histories of those traveling through the crystal and their deposited energies were logged. Energy-window weighting was performed with a gaussian filtered energy window to implement the

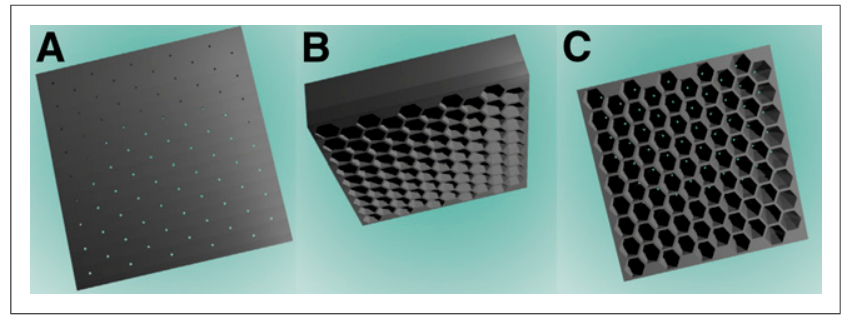

FIGURE 1. Rendering of PC collimator from different perspectives: from face of collimator (A), side of collimator $(B)$, and back of collimator $(C)$.

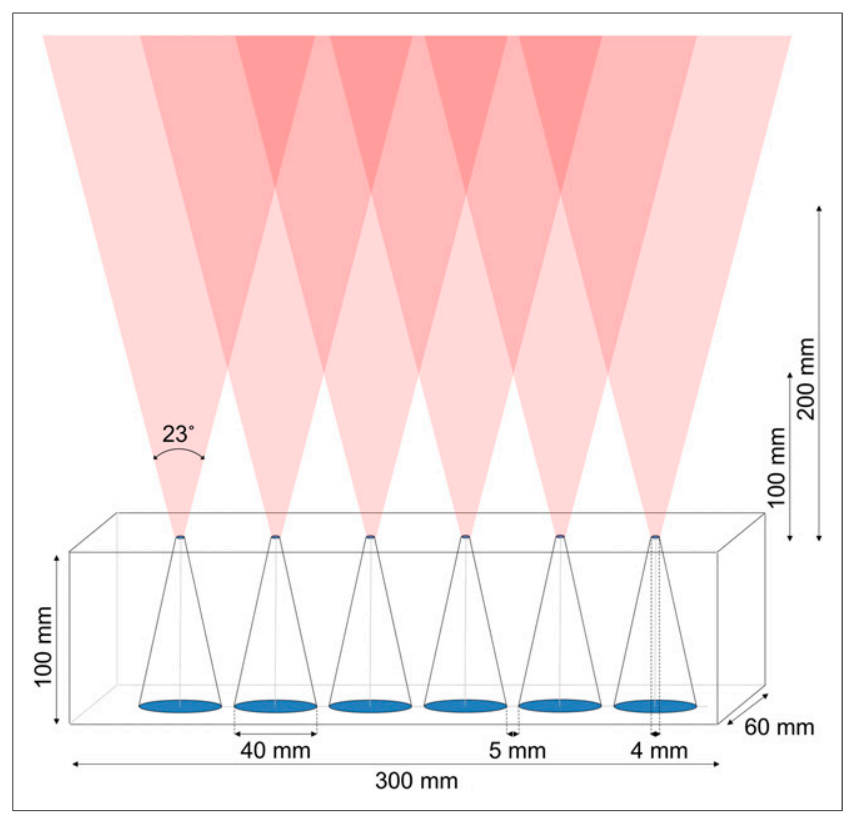

FIGURE 2. Schematic drawing of PC collimator prototype showing that FOVs of adjacent cones overlap as distance from collimator increases. Darker colors mark areas that are imaged by multiple cones. FOVs of adjacent cones overlap at distances greater than $100 \mathrm{~mm}$ ( 2 cones) and $200 \mathrm{~mm}$ ( 3 cones) from collimator surface.

energy resolution. The intrinsic spatial resolution was modeled by applying gaussian convolution. The gamma camera was simulated including the $9.5-\mathrm{mm} \mathrm{NaI}$ crystal, aluminum crystal housing, lead camera housing, and photomultiplier tube crown glass acting as backscatter material, as described and validated previously by our group $(6,15,16)$.

${ }^{99 \mathrm{~m} T c},{ }^{131} \mathrm{I}$, and ${ }^{18} \mathrm{~F}$ point sources were simulated to compare the prototype PC collimator with the LEHR and HEGP collimators. First, simulations of 2 point sources in air placed $2 \mathrm{~cm}$ apart at 5-, 10-, 15-, and $20-\mathrm{cm}$ distances from the collimator face were performed to study the capability of the collimator to spatially separate the signal of the 2 sources at varying distances (Supplemental Fig. 2A). Second, simulations of a single point source in air placed $10 \mathrm{~cm}$ from the collimator face were done to study the PSF broadening (Supplemental Fig. 2B). Contributions of primary photons, penetrated photons, and other nonprimary photons, such as scattered photons, to the total PSF were analyzed separately. One-dimensional profiles were created from the 2-dimensional (2D) detector data by summation over a width of $4 \mathrm{~cm}$ (Supplemental Fig. 3). The full width at half maximum (FWHM) and full width at tenth maximum (FWTM) were calculated directly from the acquired profiles. The area under the curve of the measured profiles was determined as a measure of sensitivity, in addition to the maximum value of the profiles.

\section{Phantom Experiments}

Phantom. A flangeless Esser phantom (Data Spectrum Corp.) with a lid holding refillable thin-walled cylinders of $8,12,16$, and $25(\times 3)$ $\mathrm{mm}$ in diameter and a solid Teflon (DuPont) cylinder of $25 \mathrm{~mm}$ in diameter was used to quantitatively evaluate the image quality of the different collimators. The phantom inner diameter is $204 \mathrm{~mm}$, the inner phantom height is $186 \mathrm{~mm}$, and the inner cylinder height is $38.1 \mathrm{~mm}$. Table 1 shows the activity concentrations in the hot cylinders and the background of the phantom for the different isotope experiments. As shown in Supplemental Figure 4, the 3 cold cylinders contained Teflon, air, and water. SPECT acquisitions of the same phantom using the PC and HEGP or LEHR collimators were performed successively to compare phantom measurements with the same activity concentration ratio. 
TABLE 1

Overview of Activity Concentration and Ratios Used During Phantom Experiments

\begin{tabular}{lcccc}
\hline & & \multicolumn{2}{c}{ Activity concentration $(\mathrm{MBq} / \mathrm{mL})$} \\
\cline { 3 - 4 } Isotope & Photon energy $(\mathrm{keV})$ & Hot cylinders & Background \\
\hline $99 \mathrm{mTC}$ & 141 & 0.88 & 0.08 & 11.4 \\
$131 \mathrm{I}$ & $364,637,284$ & 0.66 & 0.06 & 10.4 \\
$18 \mathrm{~F}$ & 511 & 1.34 & 0.12 \\
\hline
\end{tabular}

Scanners and Acquisition. A dual-head Symbia T16 (Siemens Healthcare) SPECT/CT system with LEHR $\left({ }^{99 \mathrm{~m} T c)}\right.$ and HEGP $\left({ }^{131} \mathrm{I}\right.$ and ${ }^{18} \mathrm{~F}$ ) collimators was used to acquire SPECT images on a $128 \times$ 128 grid with a $2.40 \times 2.40 \mathrm{~mm}$ pixel size. The PC collimator was mounted on a single-head Argus Epic (ADAC Laboratories) gamma camera, which acquired images on a $256 \times 256$ grid with a $2.32 \times 2.32$ $\mathrm{mm}$ pixel size. Both systems use a $9.5-\mathrm{mm} \mathrm{NaI}(\mathrm{Tl})$ scintillation crystal. Because the construction holding the PC collimator restricted rotation of the gantry, a computer-controlled stepper motor was used to accurately rotate the phantom (Fig. 3). The computer controlling the phantom rotation was also used to start gamma-camera acquisitions, to synchronize phantom rotation with image acquisition. The energy window width around the photopeak was $15 \%$ for ${ }^{99 \mathrm{~m}} \mathrm{Tc}$, $15 \%$ for ${ }^{131} \mathrm{I}$, and $20 \%$ for ${ }^{18} \mathrm{~F}$. One hundred twenty projections were acquired over a $360^{\circ}$ orbit for all experiments, and the distance from the center of rotation to the collimator face was $15 \mathrm{~cm}$. The acquisition time was $30 \mathrm{~s}$ per view or proportionally longer to correct for the decay time between consecutive measurements of the same phantom.

Reconstruction. Iterative 2D maximum-likelihood expectation maximization reconstruction algorithms incorporating attenuation correction and resolution recovery in the forward and backward projectors were used for image reconstruction (17). Reconstructions of a single (2D) slice instead of a (3-dimensional) volume were performed, because the prototype PC collimator consists of only a single row of cones. The measured projections of the Siemens Symbia T16 system were acquired with parallel-hole collimators, and images were reconstructed using an in-house-developed 2D maximum-likelihood expectation maximization algorithm based on a rotation-based projector/backprojector pair. Convolution with depth-dependent gaussian PSFs was applied to achieve resolution recovery in both

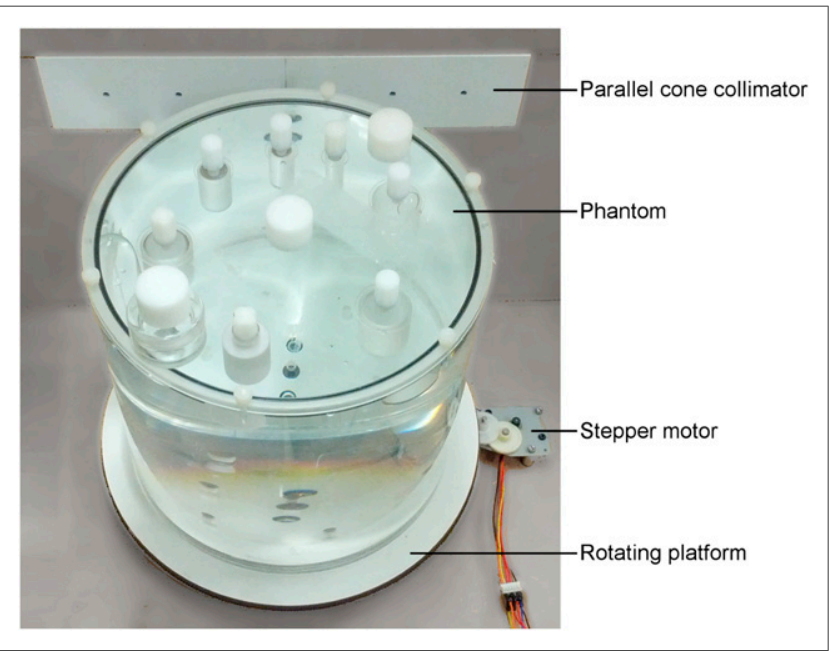

FIGURE 3. Picture of experimental setup showing PC collimator, phantom, stepper motor controlling phantom rotation, and rotating platform. forward and backward projections, including modeling of septal penetration by taking into account the effective septal length. The projections of the ADAC Argus Epic gamma camera were acquired with the PC collimator, and images were reconstructed using a voxeldriven 2D maximum-likelihood expectation maximization algorithm estimating the contribution of voxels covered by the geometry of the cone FOV by modeling the point response geometrically $(18,19)$. The finite dimensions of the cone opening and collimator penetration were considered to incorporate resolution recovery (20). To prevent the occurrence of multiplexing artifacts, no backprojection was performed of pixels that contained information from multiple cones, that is, the area between adjacent cones in which overlap occurs most likely due to the limited intrinsic spatial resolution of the Anger-logic position estimation $(21,22)$. Other potential causes for overlap of adjacent pinhole projections are unlikely, because the crystal-collimator geometry does not allow for overlap of primary photons from adjacent cones, the multiplexing is observed even at low energies for which collimator penetration is negligible, and the Monte Carlo simulations show collimator scatter is negligible. No scatter correction, smoothing, or filtering was applied during or after reconstruction to ensure that the reconstruction algorithms for the PC and parallel-hole collimators incorporated the same features.

Quantitative Analysis. Supplemental Figure 4 shows a schematic drawing of the phantom overlaid with the positions of the hot cylinder, cold cylinder, and background regions of interest (ROIs). To quantitatively evaluate the quality of reconstructed images, several image quality measures were calculated. Wherever possible, the 2007 guidelines of National Electrical Manufacturers Association were adopted (23). Contrast recovery coefficients (CRCs) were calculated for each of the hot cylinders using the following equation:

$$
\mathrm{CRC}=\frac{C_{H} / C_{B}-1}{R-1} \times 100 \%,
$$

where $C_{H}$ is the mean pixel value in the hot cylinder ROI under consideration, $C_{B}$ is the mean pixel value in the background ROIs, and $R$ is the actual activity concentration ratio between hot cylinders and background. Assuming that $R$ is zero for cold ROIs, cold CRCs are calculated using

$$
\mathrm{CRC}=\left(1-\frac{C_{C}}{C_{B}}\right) \times 100 \%,
$$

where $C_{C}$ is the mean pixel value in the cold cylinder ROI. To assess the noise in the reconstructed data, the coefficient of variation (CV) in the pixels contained by the background ROIs shown in Supplemental Figure 4 is calculated using:

$$
\mathrm{CV}=\frac{S T D V_{B}}{C_{B}} \times 100 \%
$$

where $S T D V_{B}$ is the SD of the pixel values in all background ROIs. The ROI analysis took into account partial pixels by applying weights 


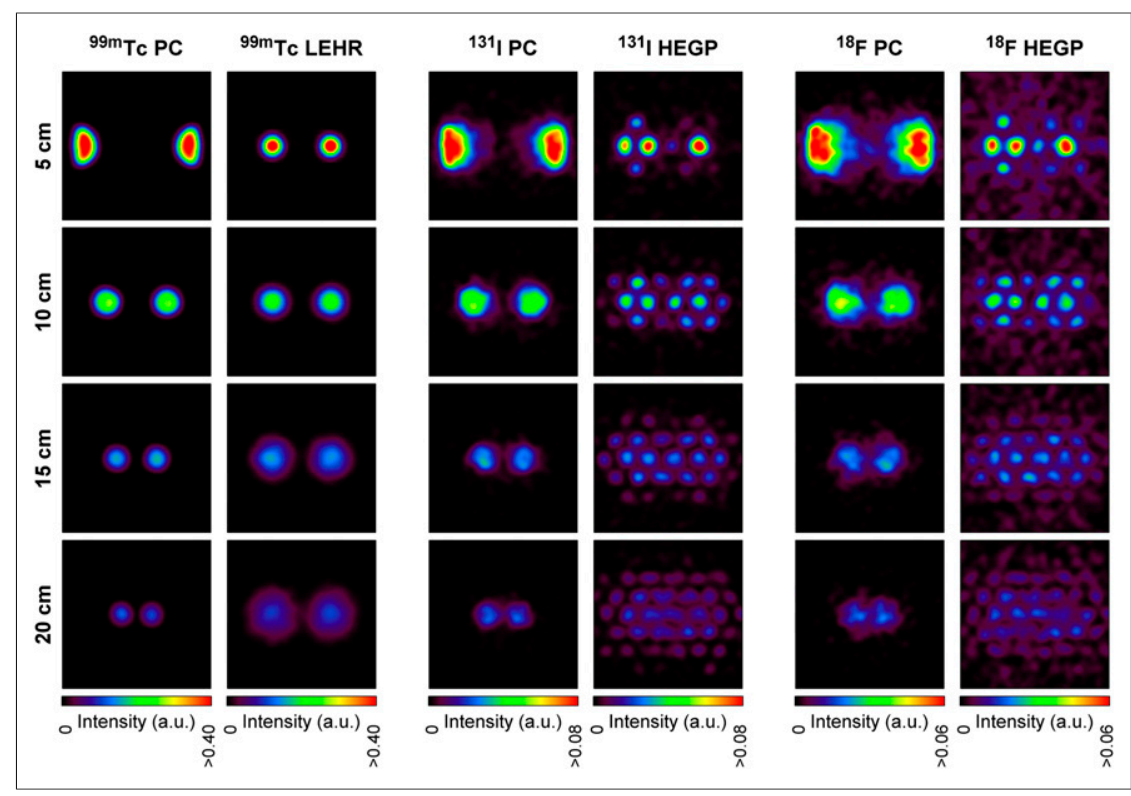

FIGURE 4. Double-point-source Monte Carlo simulations of PC, LEHR, and HEGP collimators for ${ }^{99 \mathrm{mTC}}{ }^{131} \mathrm{I}$, and ${ }^{18} \mathrm{~F}$, respectively. a.u. = arbitrary units.

to the pixels on the edge of the ROI based on the fraction of their surface that fell within the circular ROI. The position of each ROI was determined with subpixel resolution such that the sum of CRCs was maximized. The diameter of the ROIs was taken equal to the inner diameter of cylinders.

The error of the mean value in the hot and background ROIs was determined with $95 \%$ confidence intervals, and error propagation was used to calculate the uncertainties in the CRCs (24). The Standard Equivalency Test was used to determine whether or not measured CRCs were significantly different, described mathematically by

$$
|a-b| \leq 2 \sqrt{\sigma_{a}^{2}+\sigma_{b}^{2}}
$$

Eq. 4

where $a$ and $b$ are values measured with uncertainties $\sigma_{a}$ and $\sigma_{b}$, respectively.

\section{RESULTS}

\section{Double-Point-Source Simulations}

Figure 4 shows the Monte Carlo-simulated count distributions of 2 point sources placed $2 \mathrm{~cm}$ apart at several distances from the collimator face. Both the LEHR and the PC collimator enable detection of the two ${ }^{99 \mathrm{~m}} \mathrm{Tc}$ sources separately at all simulated distances. However, for ${ }^{131} \mathrm{I}$ and ${ }^{18} \mathrm{~F}$ point sources at distances greater than $10 \mathrm{~cm}$, a difference can be seen in the level of point source separation between the PC and the HEGP collimator. The simulations of the PC collimator show the typical magnification effect expected for the cone geometry; the projected maxima are further apart when the simulated point sources are close to the collimator face and vice versa. The FOV of adjacent cones is overlapping for distances more than $10 \mathrm{~cm}$ from the collimator face, and sources can be imaged by multiple cones in this region. Hence, the sensitivity of the collimator is not a continuous function of the distance to the collimator face. The hexagonal pattern of collimator septa can be observed in the simulations of the HEGP collimator, because of the relatively large hole sizes and thick septa.

\section{Single-Point-Source Simulations}

The PSF simulations of ${ }^{131} \mathrm{I}$ and ${ }^{18} \mathrm{~F}$ show that PSF broadening is largely caused by septal penetration and that this effect is more clearly present with the HEGP collimator than with the PC collimator (Fig. 5; Table 2). The width of the FWHM of primary photons as measured by the PC collimator is nearly constant for all isotopes, despite the small variations caused by the energy-dependent intrinsic spatial resolution. For ${ }^{131} \mathrm{I}$ and ${ }^{18} \mathrm{~F}$, the FWHM and FWTM of the total PSF measured by the HEGP collimator are higher than the values measured by the PC collimator. Furthermore, the shape of the distribution of penetrated photons is different for the PC and HEGP collimators. For the HEGP collimator, the positions of the septa cause variations in the distribution of penetrated photons. For the PC collimator, 2 maxima are observed in the distribution of pene-

trated photons where the edges of the cone
FIGURE 5. Profiles $(4 \mathrm{~cm}$ wide) through Monte Carlo-simulated projection images of single point source at $10 \mathrm{~cm}$ from collimator face. Graphs are shown for PC collimator (A-C), LEHR collimator (D), and HEGP collimator (E and F) for ${ }^{99 m T c}$, ${ }^{131} \mathrm{I}$, and ${ }^{18} \mathrm{~F}$. 
TABLE 2

FWHM and FWTM of Total PSF and of Primary Photon Profiles Shown in Figure 5

\begin{tabular}{|c|c|c|c|c|c|}
\hline Isotope & Collimator & $\begin{array}{l}\text { FWHM } \\
\quad(\mathrm{cm})\end{array}$ & $\begin{array}{l}\mathrm{FWHM}_{\mathrm{pri}} \\
\quad(\mathrm{cm})\end{array}$ & $\begin{array}{c}\text { FWTM } \\
\text { (cm) }\end{array}$ & $\begin{array}{c}\text { FWTM } \\
\text { (cmi }\end{array}$ \\
\hline \multirow[t]{2}{*}{ 99mTc } & PC & 0.7 & 0.7 & 1.2 & 1.2 \\
\hline & LEHR & 0.8 & 0.7 & 1.4 & 1.3 \\
\hline \multirow[t]{2}{*}{$131 \mid$} & PC & 0.9 & 0.7 & 1.7 & 1.3 \\
\hline & HEGP & 1.4 & 1.2 & 2.8 & 2.3 \\
\hline \multirow[t]{2}{*}{${ }^{18} \mathrm{~F}$} & PC & 1.1 & 0.7 & 2.1 & 1.4 \\
\hline & HEGP & 1.6 & 1.3 & 5.5 & 2.5 \\
\hline
\end{tabular}

$\mathrm{FWHM}_{\text {tot }}=\mathrm{FWHM}$ of total PSF; FWHM $\mathrm{Fri}=\mathrm{FWHM}$ of primary PSF; FWTM tot $=$ FWTM of total PSF; FWTM pri $=$ FWTM of primary PSF.

are situated. This is where penetration is likely to occur more frequently due to the limited thickness of the cone edges.

Although the FWHM of the primary PSF for ${ }^{99 \mathrm{~m} T c}$ is equal for both collimators, the FWTM of the total PSF is slightly larger for the LEHR collimator, because of broadening by septumpenetrated and collimator-scattered photons.

The sensitivity, measured over the profiles as the total amount of counts per decay (Fig. 5), was found to be higher for the LEHR and HEGP collimators than for the PC collimator. However, the maximum value of the profiles was higher for the PC collimator (Table 3).

\section{Phantom Experiments}

Two-dimensional reconstructed images were created comparing the PC collimator with the LEHR and HEGP collimators (Supplemental Fig. 5). To evaluate the image quality quantitatively, CRCs and noise measures are shown as a function of the number of iterations (Supplemental Fig. 6). For comparison, images after 37, 43, and 57 iterations for the PC and 49, 122, and 145 iterations for the parallel-hole collimators for ${ }^{99 \mathrm{~m}} \mathrm{Tc},{ }^{131} \mathrm{I}$, and ${ }^{18} \mathrm{~F}$, respectively, are shown, because these have identical background noise levels (CV) of $15 \%$. These same numbers of iterations were used to quantitatively compare CRCs between collimators at equal noise levels, as shown in Figure 6. When the Standard Equivalency Test with a $95 \%$ confidence interval was used, the PC hot CRCs were not statistically different from the LEHR CRCs for ${ }^{99 \mathrm{~m} T c}$, whereas PC cold CRCs were significantly lower than those of the LEHR. For ${ }^{131} \mathrm{I}$, the hot CRCs of the PC were significantly higher than those of the HEGP. The Teflon cold CRC was significantly higher for the PC collimator, whereas the cold CRC with air was significantly higher for the parallel-hole collimator. No significant difference was observed for the cold CRC with water. For ${ }^{18} \mathrm{~F}$, all CRCs obtained with the PC collimator were significantly higher than those of the parallel-hole collimator, except for the cold CRC with air. In general, the CRCs decreased with increasing photon energy.

\section{DISCUSSION}

In this study, we have presented a PC collimator as an alternative to the customary parallel-hole collimators for high-energy photon SPECT imaging.
Monte Carlo simulations and phantom measurements were performed to compare the PC collimator with LEHR and HEGP parallel-hole collimators. The Monte Carlo simulations showed that PSF broadening is largely caused by septal penetration of the HEGP collimator and that this effect is reduced by the PC collimator. The phantom experiments demonstrated that the $2 \mathrm{D}$ PC collimator can be used for low- and high-energy SPECT. It was found that, at equal noise level, the CRCs obtained by the PC collimator were similar to those of the LEHR collimator for ${ }^{99 \mathrm{~m}} \mathrm{Tc}$ and on average higher than those of the HEGP collimator for ${ }^{131} \mathrm{I}$ and ${ }^{18} \mathrm{~F}$.

The total amount of counts over the profiles was calculated for single-point-source simulations as a measure of sensitivity. In this study, a distance of $10 \mathrm{~cm}$ was used, so that the magnification factor was approximately 1 , for both the PC collimator and the parallel-hole collimators. Because the sensitivity is highest for the PC collimator when the point source is positioned directly in front of the cone, this might not be a fair comparison with the parallel-hole collimators. On the other hand, the measurements do not take into account that a point source is possibly imaged by multiple cones. In general, it is difficult to define a single metric to assess the impact of the sensitivity on image quality. Therefore, the CRCs and $\mathrm{CV}$ in reconstructed images were studied.

Because of the design of the collimator, the acquisition of a single planar projection does not directly yield a useful image. Sampling of the entire FOV is achieved by performing tomography, and useful images are generated after reconstruction. The sensitivity of the collimator depends on by how many cones the source is seen and is therefore not a continuous function of the distance to the collimator face. The use of multiple cones ensures that the sensitivity does not decrease as rapidly with distance from the collimator as with single-pinhole collimators.

The error bars presented in Figure 6 are based on the noise level of the image, which means that they are a measure of (pixel) precision but not of accuracy. Therefore, the error bars only represent random errors and do not include possible systematic errors. For example, measurement errors in the activity concentration might have yielded a systematic over- or underestimation of the background-to-cylinder ratio. However, such a systematic misestimation would have affected CRC quantification of the parallelhole and PC collimator proportionally, because measurements of an isotope with different collimators were performed consecutively using the same phantom. Consequently, the validity of relative image quality differences that were observed between the

TABLE 3

Total and Maximum Amount of Counts per Decay of Profiles Shown in Figure 5

\begin{tabular}{cccc}
\hline Isotope & Collimator & $\begin{array}{c}\text { Profile area } \\
\text { (counts/decay) }\end{array}$ & $\begin{array}{c}\text { Profile maximum } \\
\text { (counts/decay) }\end{array}$ \\
\hline${ }^{99 m T C}$ & PC & $8.8 \times 10^{-5}$ & $1.2 \times 10^{-5}$ \\
& LEHR & $9.1 \times 10^{-5}$ & $1.1 \times 10^{-5}$ \\
${ }^{131}$ I & PC & $2.9 \times 10^{-5}$ & $2.9 \times 10^{-6}$ \\
& HEGP & $3.1 \times 10^{-5}$ & $2.0 \times 10^{-6}$ \\
${ }^{18} \mathrm{~F}$ & PC & $4.7 \times 10^{-5}$ & $3.8 \times 10^{-6}$ \\
& HEGP & $6.6 \times 10^{-5}$ & $3.1 \times 10^{-6}$ \\
\hline
\end{tabular}



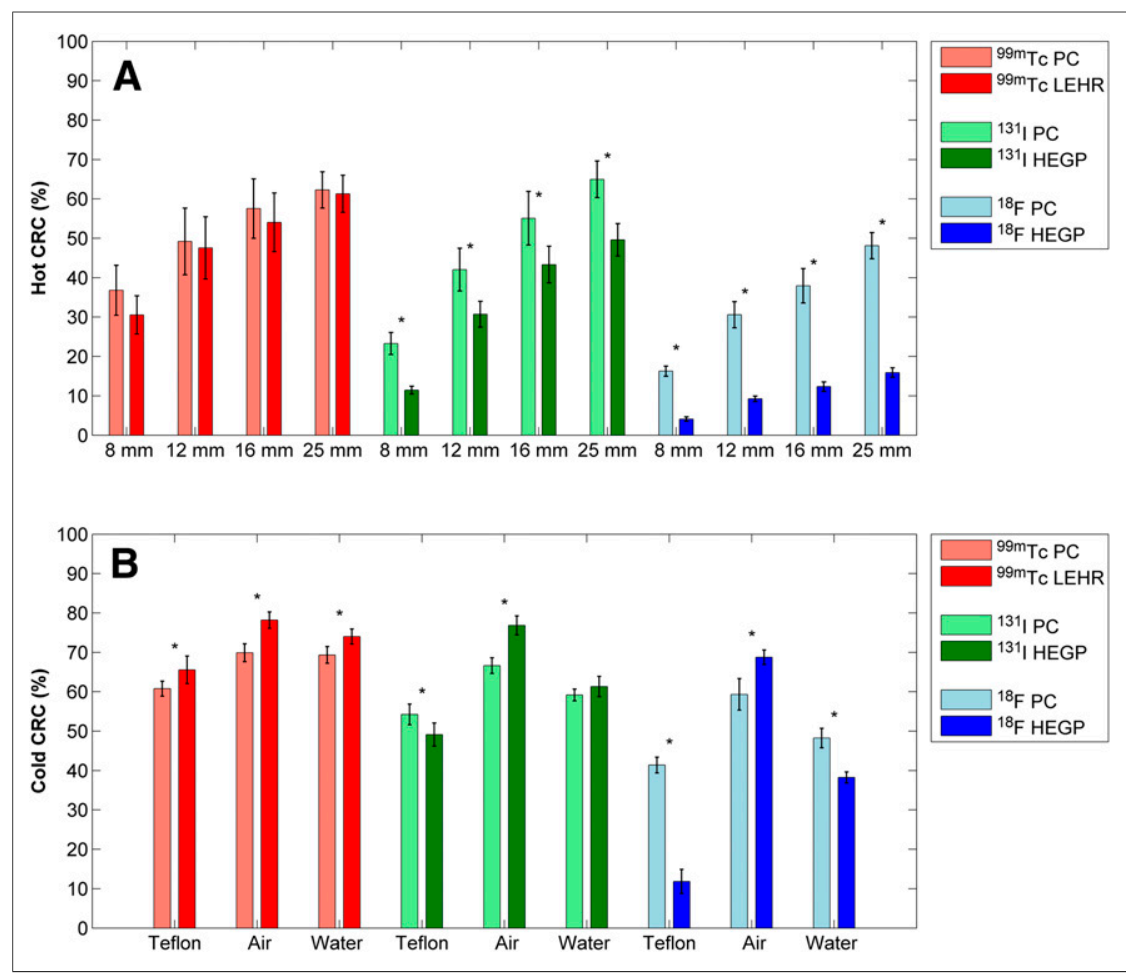

FIGURE 6. CRCs of images at identical noise levels (CV $=15 \%)$ for $P C$ and parallel-hole collimators shown for hot cylinders (A) and cold cylinders (B) for ${ }^{99 m T c},{ }^{131} \mathrm{I}$, and ${ }^{18} \mathrm{~F}$. ${ }^{*}=$ significantly different CRCs. collimator can improve image quality in clinical practice for high-energy applications.

\section{CONCLUSION}

A PC collimator for high-energy SPECT imaging has been designed and evaluated. Monte Carlo simulations show less broadening of the PSF due to collimator penetration for the proposed collimator than for a high-energy parallel-hole collimator. Additionally, phantom measurements with a single-slice prototype of the proposed PC collimator have shown the potential for significantly improved quality of high-energy SPECT images in comparison with standard parallel-hole collimators.

\section{DISCLOSURE}

The costs of publication of this article were defrayed in part by the payment of page charges. Therefore, and solely to indicate this fact, this article is hereby marked "advertisement" in accordance with 18 USC section 1734. No potential conflict of interest relevant to this article was reported. collimator types is not compromised by neglecting these potential systematic errors. However, such errors might explain the better 25-mm cylinder CRC for ${ }^{131} \mathrm{I}$ as compared with ${ }^{99 \mathrm{~m}} \mathrm{Tc}$, although the observed difference is not significant.

The prototype PC collimator consists of a single row of cones to simplify production and to keep costs low. Consequently, SPECT data could be reconstructed only to 2D slices (instead of 3-dimensional volumes) for the PC collimator. The SPECT data for the parallel-hole collimators were also reconstructed in 2 dimensions, to ensure that collimator differences were studied rather than differences in the reconstruction algorithm.

To prevent the occurrence of multiplexing artifacts, 2 pixels on either side of the cone were not used for backprojection, that is, 4 of approximately 20 pixels per cone. For future research, it should be considered that the relative amount of masked pixels is larger in 2D projections than in 1-dimensional projections. However, the amount of masked pixels can possibly be reduced, because no fundamental need for this masking exists, by optimizing the collimator design, for example, by optimizing the thickness of septa at the bottom of the cones.

This study shows that the image quality of the PC collimator decreases as the photon energy increases but that this effect is not as severe as observed for the parallel-hole collimator, indicating that the PC collimator could be used for a variety of radioisotopes and photon energies. Using a single collimator for multiple purposes has many benefits in clinical practice, because changing collimators between examinations is time consuming and requires large and heavy collimator carts. A study comparing acquisitions from full-FOV collimators with full-3D acquisitions and algorithms may give conclusive answers as to whether the PC

\section{ACKNOWLEDGMENT}

We thank Gert Nouwen for technical support.

\section{REFERENCES}

1. Avram AM. Radioiodine scintigraphy with SPECT/CT: an important diagnostic tool for thyroid cancer staging and risk stratification. J Nucl Med Technol. 2014;42:170-180.

2. Dewaraja YK, Frey EC, Sgouros G, et al. MIRD pamphlet no. 23: quantitative SPECT for patient-specific 3-dimensional dosimetry in internal radionuclide therapy. $J$ Nucl Med. 2012;53:1310-1325.

3. Shcherbinin S, Grimes J, Bator A, Cwikla JB, Celler A. Three-dimensional personalized dosimetry for 188 Re liver selective internal radiation therapy based on quantitative post-treatment SPECT studies. Phys Med Biol. 2013; 59:119-134.

4. Vente MAD, Wondergem M, Tweel I, et al. Yttrium-90 microsphere radioembolization for the treatment of liver malignancies: a structured meta-analysis. Eur Radiol. 2009;19:951-959.

5. Dewaraja YK, Ljungberg M, Koral KF. Characterization of scatter and penetration using Monte Carlo simulation in ${ }^{131} \mathrm{I}$ imaging. J Nucl Med. 2000; 41:123-130.

6. Elschot M, Nijsen JFW, Dam AJ, de Jong HWAM. Quantitative evaluation of scintillation camera imaging characteristics of isotopes used in liver radioembolization. PLoS ONE. 2011;6:e26174.

7. Walrand S, Hesse M, Demonceau G, Pauwels S, Jamar F. Yttrium-90-labeled microsphere tracking during liver selective internal radiotherapy by bremsstrahlung pinhole SPECT: feasibility study and evaluation in an abdominal phantom. EJNMMI Res. 2011;1:32.

8. van Lingen A, Huijgens $\mathrm{P}$, Visser F, et al. Performance characteristics of a 511-keV collimator for imaging positron emitters with a standard gamma-camera. Eur $J$ Nucl Med. 1992;19:315-321.

9. Dewaraja YK, Ljungberg M, Koral KF. Accuracy of ${ }^{131}$ I tumor quantification in radioimmunotherapy using SPECT imaging with an ultra-high-energy collimator: Monte Carlo study. J Nucl Med. 2000;41:1760-1767. 
10. Wanet PM, Sand A, Abramovici J. Physical and clinical evaluation of highresolution thyroid pinhole tomography. J Nucl Med. 1996;37:2017-2020.

11. Nuyts J, Vunckx K, Defrise M, Vanhove C. Small animal imaging with multipinhole SPECT. Methods. 2009;48:83-91.

12. Symbia TruePoint SPECT $\bullet C T$. Siemens website. http://usa.healthcare.siemens.com/siemens_hwem-hwem_ssxa_websites-context-root/wcm/idc/siemens_ hwem-hwem_ssxa_websites-context-root/wcm/idc/groups/public/@us/documents/ download/mdaw/nde2/ edisp/reju_spectct_spec_sheet-00304913.pdf. Accessed January 22, 2015.

13. Hendricks JS, McKinney GW, Waters LS, et al. MCNPX Extensions Version 2.5.0. Technical report LA-UR-04-0570. Los Alamos, NM: Los Alamos National Laboratory; 2004.

14. Khazov Y, Mitropolsky I, Rodionov A. Nuclear Data Sheets for A $=131$. Nucl Data Sheets. 2006;107:2715-2930.

15. Elschot M, Lam MGEH, van den Bosch MAAJ, Viergever MA, de Jong HWAM. Quantitative Monte Carlo-based ${ }^{90}$ Y SPECT reconstruction. J Nucl Med. 2013; 54:1557-1563.

16. Elschot M, Smits MLJ, Nijsen JFW, et al. Quantitative Monte Carlo-based holmium166 SPECT reconstruction. Med Phys. 2013;40:112502.

17. Shepp LA, Vardi Y. Maximum likelihood reconstruction for emission tomography. IEEE Trans Med Imaging. 1982;1:113-122.
18. Bal G, Acton PD. Analytical derivation of the point spread function for pinhole collimators. Phys Med Biol. 2006;51:4923-4950.

19. Metzler SD, Bowsher JE, Greer KL, Jaszczak RJ. Analytic determination of the pinhole collimator's point-spread function and RMS resolution with penetration. IEEE Trans Med Imaging. 2002;21:878-887.

20. Vanhove C, Andreyev A, Defrise M, Nuyts J, Bossuyt A. Resolution recovery in pinhole SPECT based on multi-ray projections: a phantom study. Eur J Nucl Med Mol Imaging. 2007;34:170-180.

21. Min BJ, Choi Y, Lee N-Y, et al. Unmatched projector/backprojector pair for demultiplexing in multipinhole emission computed tomography. Opt Eng. 2010;49:127004-1-127004-9.

22. Vunckx K, Suetens P, Nuyts J. Effect of overlapping projections on reconstruction image quality in multipinhole SPECT. IEEE Trans Med Imaging. 2008;27:972-983

23. Perkins A, Stearns C, Chapman J, Kolthammer J, Williams JJ, Casey M. National Electrical Manufacturers Association (NEMA). NEMA Standards Publication NU 2-2007 Performance Measurements of Positron Emission Tomographs. Rosslyn, VA: NEMA; 2007.

24. Taylor JR. An Introduction to Error Analysis: The Study of Uncertainties in Physical Measurements. Sausalito, CA: University Science Books; 1997: 73-79. 